\title{
Exosome-derived miR-196b-5p facilitates intercellular interaction in infantile hemangioma via down-regulating CDKN1B
}

\author{
Qi-Zhang Wang, Ze-Liang Zhao, Chao Liu, Jia-Wei Zheng \\ Department of Oromaxillofacial Head and Neck Oncology, Shanghai Ninth People's Hospital, College of Stomatology, Shanghai Jiao Tong \\ University School of Medicine, Shanghai, China \\ Contributions: (I) Conception and design: QZ Wang, ZL Zhao; (II) Administrative support: JW Zheng, C Liu; (III) Provision of study materials \\ or patients: QZ Wang; (IV) Collection and assembly of data: QZ Wang, C Liu; (V) Data analysis and interpretation: QZ Wang, ZL Zhao; (VI) \\ Manuscript writing: All authors; (VII) Final approval of manuscript: All authors \\ Correspondence to: Jia-Wei Zheng, DDS, PhD. Department of Oromaxillofacial Head and Neck Oncology, Shanghai Ninth People's Hospital, College \\ of Stomatology, Shanghai Jiao Tong University School of Medicine, No. 639 Zhizaoju Road, Shanghai 200011, China. \\ Email: davidzhengjw@sjtu.edu.cn.
}

\begin{abstract}
Background: Though infantile hemangioma (IH) is a common benign vascular tumor, its pathogenesis remains unclear. This study explored the function of hemangioma-derived stem cells (HemSCs) derived exosomes, which exerted an intercellular effect on hemangioma-derived endothelial cells (HemECs).

Methods: First, HemSCs and HemECs were extracted and cultured. HemSCs derived exosomes (HemSCsexos) were harvested. miRNA sequencing and target prediction were used to explore differentially expressed miRNAs and potential binding targets. After HemECs were co-cultured with HemSCs-exos, a series of in vitro assays were then performed including cell counting kit-8 (CCK-8) assay, cell apoptosis assay, cell cycle assay and tube formation assay to evaluate proliferation, angiogenesis abilities, etc. qRT-PCR and Western blot were conducted to detect the expression level of target genes and proteins.

Results: After co-culturing with HemSCs-exos, proliferation, and angiogenesis abilities of HemECs were enhanced, while apoptosis and cell cycle arrest rate were decreased. MiR-196b-5p was observed to be significantly highly expressed in HemSCs-exos. CDKN1B was identified as the binding target of miR-196b5 p. HemECs' proliferation and angiogenesis abilities were elevated when co-cultured with exosomes from HemSCs transfected with miR-196b-5p mimic. In addition, apoptosis rate declined, and lower cells were arrested in G0/G1 phases. Cyclin E, bcl-2 were significantly highly expressed, whereas p27, Bax expression were significantly down-regulated. The positive effect of miR-196b-5p in HemSCs-exos was dramatically reversed when HemECs were transfected with oe-CDKN1B.

Conclusions: The current study found a novel intercellular interaction between IH cells. Briefly, exosomederived miRNA-196b-5p in HemSCs could facilitate proliferation and angiogenesis abilities, and attenuate apoptosis and cell cycle repression rate of HemECs by directly binding with CDKN1B.
\end{abstract}

Keywords: Infantile hemangioma (IH); exosome; miRNA-196b-5p; CDKN1B; cell cycle

Submitted Sep 16, 2020. Accepted for publication Dec 11, 2020.

doi: 10.21037/atm-20-6456

View this article at: http://dx.doi.org/10.21037/atm-20-6456

\section{Introduction}

Infantile hemangioma $(\mathrm{IH})$ is the most common benign vascular tumor of infancy (1), affecting nearly $3-10 \%$ of infants (2). The incidence rises to $22-30 \%$ in premature or low-birthweight infants. In most cases, IH evolution can be divided into 3 phases: proliferating phase (approximately age $0-1$ ), involuting phase (age 1-5), and involuted phase (age 5-10) (3). Despite its spontaneous involution, IH can cause disfigurement or organ dysfunction in many children, along with potential psychological effects and economic 
burdens.

Regarding the pathogenesis of $\mathrm{IH}$, the exact mechanism remains unclear. Khan et al. (4) reported that $\mathrm{IH}$ originated from CD133+ hemangioma-derived stem cells (HemSCs). Previous studies have repeatedly emphasized that the existence of HemSCs are necessary for IH formation $(4,5)$. HemSCs function as stem cells. They can differentiate into hemangioma-derived endothelial cells (HemECs), pericytes, and adipocytes in the involuted phase $(6,7)$, playing a pivotal role in the whole IH progression. In addition, previous studies documented that HemSCs could interact with other IH cells. HemSCs regulated blood vessel formation via the expression of vascular endothelial growth factor-A (VEGF-A) (8). VEGF-A bound with vascular endothelial growth factor receptor 2 (VEGFR2), which was constitutively activated among HemECs (9). Conversely, it was reported that HemECs affected the biological viability of HemSCs alike. Smadja et al. (10) discovered that HemECs in proliferating phase IH expressed E-selectin, stimulating the migration and adhesion of HemSCs. E-selectin expression would be enhanced when HemECs were exposed to VEGF-A.

Extracellular vesicles (EVs) released by cells participate in the process of intercellular communication and material exchange in the tumor microenvironment (TME). Exosomes are specific subtype of EVs characterized by a size of 40-150 $\mathrm{nm}$ in diameter and cup-shaped microvesicles under an electron microscope $(11,12)$. Exosomes biogenesis relies on the endocytic pathway (13). Briefly, they originate from endosomal packaging into multivesicular bodies (MVBs). Then, exosomes are generated inside MVBs and released extracellularly after MVBs fuse with the plasma membrane. Though the detailed mechanism has not been well characterized, the process of exosomes formation is driven by the endosomal sorting complex required for transport (ESCRT) as described previously (14). Exosomes contain distinct bioactive materials such as RNA, DNA, lipids, proteins (15) enclosed by cholesterol-rich membrane. Secreted by most cells, exosomes can target neighboring and distant cells by trafficking in TME and circulation. Recipient cells selectively internalize exosomes via exosomal specific markers such as CD63. Therefore, exosomes have a significant role in regulating several physiological or pathological processes (16). Though, according to previous studies, there was no direct correlation between IH and exosomes, emerging evidence suggested that exosomes secreted by endothelial cells participated in the endothelium regulation in various tissues (17-20). Moreover, it was proved that components in tumors' exosomes could regulate angiogenesis (21). Since IH is mainly composed of atypical endothelial cells, and angiogenesis is indispensable for IH formation (22), it is worthwhile to explore whether exosomes are involved in $\mathrm{IH}$ progression.

MicroRNAs(miRNAs) are a series of short non-coding small RNAs with an average length of 22 nucleotides (23). By binding to the 3' untranslated region (UTR) of the target mRNA after silencing complex formation $(24,25)$, miRNAs can generate translational repression or degradation at the posttranscriptional level, thus affecting distinct biological processes (26). miRNAs can be encapsulated by exosomes and then transported to target cells to promote intercellular communication (27). A growing number of exosomes-derived miRNAs have been identified to be able to participate in various processes of cancer (28), they can also promote endothelial regeneration (29-31). However, how exosomes-derived miRNAs affect the formation or development of IH remains to be further investigated.

Since the interaction between HemSCs and HemECs has not been fully discovered, the current study intended to explore the intercellular interaction and whether exosomes participated in this process. Possible findings are helpful to develop potential therapeutic targets for IH treatment.

We present the following article in accordance with the MDAR checklist (available at http://dx.doi.org/10.21037/ atm-20-6456).

\section{Methods}

\section{Cell culture and identification}

Proliferating IH tissues were obtained from Shanghai Ninth People's Hospital under a human subject protocol that was approved by the Institutional Ethics Committee (SH9H-2019TK168-1). All patients were provided with informed consent according to the Declaration of Helsinki (as revised in 2013).

HemSCs and HemECs were isolated from proliferating IH tissues as described previously (32). Briefly, IH tissues were digested with $0.2 \%$ collagenase A (Roche Diagnostics, USA) in Dulbecco modified Eagle medium (DMEM) (Hyclone, USA) for $30 \mathrm{~min}$ at $37^{\circ} \mathrm{C}$. Then, tissues were filtered through a $100-\mu \mathrm{m}$ cell strainer. Erythrocytes were lysed by $\mathrm{NH} 4 \mathrm{Cl}(0.8 \% \mathrm{NH} 4 \mathrm{Cl} / 0.1 \mathrm{mM}$ EDTA $)$ (StemCell Technologies, CAN) for $10 \mathrm{~min}$ on ice. CD133 ${ }^{+}$ cells and CD133- cells were isolated using anti-CD133coated magnetic beads (Miltenyi Biotec, USA) and cultured on fibronectin-coated plates with Endothelial 
Basal Medium (EBM-2) (Cambrex Bio Science, USA). EBM-2 was supplemented with $20 \%$ fetal bovine serum (FBS), SingleQuot (Cambrex Bio Science, USA), PSF. CD $133^{+}$and CD $133^{-}$cells were used as HemSCs and HemECs, respectively.

Human umbilical vein endothelial cells (HUVECs) were obtained from the American Type Culture Collection (ATCC) (CRL-1730, Manassas, USA) and were cultured in DMEM with 10\% FBS (Hyclone, USA) and 1\% penicillin and streptomycin (Sigma-Aldrich, USA) in a $37^{\circ} \mathrm{C}$ humidified atmosphere containing $95 \%$ air, $5 \% \mathrm{CO}_{2}$ (33).

\section{Single cell clonogenic and differentiation assay}

HemSCs were cultured and a single-cell suspension of HemSCs was diluted to 10 cells/mL and plated in each well of a 96-well plate. The cell number was calculated on day 2, 5, 7, 10, 14 after single-cell dilution. For adipogenesis, HemSCs were cultured in adipogenic media (Cyagen Biosciences, USA) for 14 days. Lipid droplets were detected with Oil-Red-O staining; For osteogenesis, HemSCs were cultured in osteogenic media (Cyagen Biosciences, USA) for 14 days. Alizarin Red S staining was used for osteogenesis analysis.

\section{Exosome isolation and identification}

After HemSCs and HUVECs reaching 70-80\% confluent, HemSCs and HUVECs were washed with phosphatebuffered saline (PBS) and cultured in EBM-2 medium with $10 \%$ exosome-depleted FBS (System Biosciences, USA) for an additional $48 \mathrm{~h}$. Cell supernatants were collected and centrifuged at $300 \mathrm{~g}$ for $5 \mathrm{~min}$ and $3,000 \mathrm{~g}$ for $15 \mathrm{~min}$ to remove cellular debris. Then, the supernatants were filtered through a $0.22-\mu \mathrm{m}$ filter (Millipore, USA). Next, the exosomes were isolated at $120,000 \mathrm{~g}$ for $60 \mathrm{~min}$. The pelleted exosomes were resuspended in PBS and repeatedly centrifuged once. Finally, the exosomes were stored at $-80^{\circ} \mathrm{C}$ for subsequent use. All procedures were performed at $4{ }^{\circ} \mathrm{C}$. Nanoparticle tracking analysis (NTA), transmission electron microscopy (TEM) and Western blot were used to identify the exosomes $(34,35)$. The size and concentration were measured by NTA software (Nanosight, v2.3, UK). The morphology of exosomes was observed under TEM (HITACHI, H-7650, Japan). The specific surface markers of exosomes including CD63, TSG101, HSP70 were analyzed by Western blot. Calnexin served as the negative control.

\section{miRNA sequencing and differentially expressed miRNA analysis}

HemSCs-exos and HUVECs-exos were harvested and the RNA in exosomes was sequenced by Novogene (Beijing, China). Briefly, the sequencing library of miRNA was prepared from total RNA by using NEBNext Small RNA Library Prep Set for Illumina (NEB, USA) according to the manufacturer's instructions. Subsequently, RNA was ligated with 5'-RNA and 3'-RNA adapters, reversely transcribed into cDNAs by M-MuLV Reverse Transcriptase (RNase $\mathrm{H}-)$ and PCR amplified. Library quality was assessed on the Agilent Bioanalyzer 2100 system using DNA High Sensitivity Chips. After quality control, the PCR products were sequenced by an Illumina Hiseq 2500/2000 platform, and 50-bp single-end reads were generated. Differentially expressed miRNAs between groups were analyzed using the DESeq $\mathrm{R}$ package (1.8.3). A corrected $\mathrm{P}$ value of 0.05 and at least 1.5 -fold change were set as the threshold for significantly differential expression. Target genes prediction was performed by RNAhybrid, miRanda and TargetScan.

\section{Dual-luciferase reporter gene assay}

The normal sequence of CDKN1B-3' UTR containing a miR-196b-5p binding site and its mutated 3' UTR sequence were synthesized by PCR amplification and integrated into luciferase pmirGLO vectors (Promega, USA), named CDKN1B-wild type (Wt) and CDKN1B-mutant (Mut), respectively. Then, they were co-transfected into HEK293T cells with miR-196b-5p mimic or negative controls. After transfection for $48 \mathrm{~h}$, cells were lysed to evaluate luciferase activity using the Dual luciferase Reporter Assay System (Promega, USA).

\section{Exosome uptake assay}

HemSCs-Exos and HUVECs-exos were stained with membrane fluorescent dye PKH26 (Sigma Aldrich, USA). Briefly, exosomes from HemSCs or HUVECs were resuspended and labeled with PKH26. Then, the exosomes were added to HemECs at the same dose for $24 \mathrm{~h}$ of incubation. Cells were washed with PBS and fixed in 4\% paraformaldehyde for $10 \mathrm{~min}$ and stained with DAPI for $5 \mathrm{~min}$ at room temperature. Cellular uptake of exosomes was observed under a laser confocal microscope $(\times 400)$ (Leica Microsystems, Germany). 


\section{qRT-PCR analysis}

Total RNA from the tissues, prepared cells was extracted using RNAiso Plus (TaKaRa, China). The obtained RNA was then reversely transcribed into cDNA by PrimeScript ${ }^{\mathrm{TM}}$ RT reagent Kit with gDNA Eraser (Perfect Real Time) (TaKaRa, China), and miRNA reverse transcription kit (Harbin Xinhai Gene Co., Ltd., China) following the manufacturer's protocols. Next, qPCR was performed using TB Green ${ }^{\circledR}$ Premix Ex Taq $^{\text {TM }}$ II (Tli RNaseH Plus) (TaKaRa, China), and the Applied Biosystems 7300 Real-Time PCR System (Applied Biosystems, USA) was employed for the examination of stem cell marker CD133, CD44, SOX2, LGR5, NANOG, and CDKN1B mRNA expression levels as well as miR-196b-5p, miR1301-3p, miR-1307-3p, miR-92b-5p, miR-155-5p, and miR-374a-3p expression level. GAPDH was used as the internal reference. For exosomes, procedures were basically identical except U6 was used as the internal reference. $2^{-\Delta \Delta \mathrm{Ct}}$ was used to analyze the relative expression levels. Primers used were synthesized by Sangon Biotech Co., Ltd (Shanghai, China). The primer sequences for miR-196b$5 \mathrm{p}, \mathrm{U6}$, CDKN1B, GAPDH were detailed as follows: miR196b-5p: F: 5'- GCGCGTAGGTAGTTTCCTGTT-3', R: 5' - AGTGCAGGGTCCGAGGTATT-3 '; U6: F: 5'-CTCGCTTCGGCAGCACA-3', R: 5'-AACGCTTCACGAATTTGCGT-3'; CDKN1B: F: 5'-AGATGTCAAACGTGCGAGTG-3', R : 5'-CCATGTCTCTGC AGTGCTTC-3'; GAPDH: F: 5'-TCATGGGTGTGAACCATGAGAA-3', R: 5'-GGCATGGACTGTGGTCATGAG-3'.

\section{Western blot analysis}

Total protein was extracted using RIPA lysis buffer (Beyotime Biotech, China) for $15 \mathrm{~min}$ at $4{ }^{\circ} \mathrm{C}$. Next, the protein concentration was analyzed by the bicinchoninic acid (BCA) protein assay (Beyotime Biotech, China). The protein was then separated by PAGE and then transferred onto a polyvinylidene difluoride (PVDF) membrane $(0.22 \mu \mathrm{m})$ (Millipore, USA). The membrane was blocked with $5 \%$ bovine serum albumin (BSA) for $1 \mathrm{~h}$ and incubated overnight at $4{ }^{\circ} \mathrm{C}$ with the following primary antibodies against: CD63 (Abcam Cat\# ab134045, RRID: AB_2800495), TSG101 (Abcam Cat\# ab125011, RRID: AB_10974262), HSP70 (Abcam Cat\# ab2787, RRID:AB_303300), cyclin E (Abcam Cat\# ab33911, RRID:AB_731787), bcl-2 (Abcam Cat\# ab32124, RRID:AB_725644), Bax (Abcam Cat\# ab32503, RRID:AB_725631), p27 (Abcam Cat\# ab32034, RRID:AB_2244732). GAPDH (Abcam Cat\# ab9485, RRID: AB_307275) was served as the internal control. The membrane was then incubated with horseradish peroxidase (HRP)-conjugated secondary antibodies (Cell Signaling Technology Cat\# 7074, RRID: AB_2099233) for $1 \mathrm{~h}$. The protein bands were visualized using SuperSignal enhanced chemiluminescence (ECL) substrate (Pierce, USA), and analyzed by Image Pro Plus 6.0 (Media Cybernetics, USA) software.

\section{Cells proliferation assay}

Cell counting kit-8 (Dojindo, USA) was used to examine cell proliferation ability. HemECs were planted into 96-well plates (5,000 cells/well). The CCK-8 reagent was added to each well at 24,48 , and $72 \mathrm{~h}$ after culturing with exosomes, followed by incubation for $1-4 \mathrm{~h}$ at $37^{\circ} \mathrm{C}$. A spectrophotometer was applied to read the $\mathrm{OD}$ values at $450 \mathrm{~nm}$.

\section{Cell apoptosis and cell cycle assay}

HemECs were cultured in 6-well plates and then cultured with exosomes derived from HemSCs or HUVECs when the density reached $10^{5}$ cells/well. After $48 \mathrm{~h}$, cells were harvested and centrifuged at $1,000 \mathrm{r}$ at $4{ }^{\circ} \mathrm{C}$, and resuspended again in $1 \times$ binding buffer. Annexin V-FITC apoptosis assay kit (Nanjing KeyGen Biotech. Co. Ltd., China) was used to analyze cell apoptosis within $15 \mathrm{~min}$ in the dark. Flow cytometry (Agilent Technology, USA) was utilized to determine the apoptotic rate.

HemECs were cultured in 6-well plates $\left(2 \times 10^{5}\right.$ cells/well $)$ and then cultured with exosomes derived from HemSCs or HUVECs. After $48 \mathrm{~h}$, the cells were digested and collected. After being fixed in $75 \%$ ethanol for $2 \mathrm{~h}$, the cells were treated with RNase A (Sigma Aldrich, USA) and stained in $500 \mathrm{~mL}$ PI (Sigma Aldrich, USA). Flow cytometry (Agilent Technology, USA) was employed to analyze the cell distribution in each phase, and cells in G0/G1, S, and G2/M phases in each group were counted.

\section{Tube formation assay}

HemECs were cultured in 96-well plates and then cultured with exosomes derived from HemSCs or HUVECs for $48 \mathrm{~h}$. Matrigel (BD Biosciences, USA) was thawed at $4{ }^{\circ} \mathrm{C}$ overnight and used to coat 96 -well plates. Then, plates were incubated at room temperature for 30-60 min. Next, 
HemECs were plated on the Matrigel $\left(10^{5}\right.$ cells/well). After incubation for $6 \mathrm{~h}$, the cells were visualized under a light microscope $(\times 100)$ to assess the capillary-like structures formation. The branch points of the formed tubes were scanned and quantified in at least 5 fields.

\section{Cell transfection}

HemSCs and HemECs were seeded into 6-well plates and grown to $60-80 \%$ in confluence for preparation. MiR196b-5p mimic, miR-196b-5p inhibitor, oe-CDKN1B and their negative controls (NC) were all procured from Genomeditech (Shanghai, China). Target plasmids and Lipofectamin2000 (Invitrogen, USA) were diluted using serum-free Opti-MEM (Gibco, USA) and sequentially mixed. The miR-196b-5p mimic, inhibitor was used at a dose of 100 pmol for miRNA up- and down-regulation. Next, the cells were transfected with the mixture and collected for the following analysis after $36-48 \mathrm{~h}$ of transfection.

\section{Statistical analysis}

All data were analyzed using SPSS 21.0 software (Chicago, USA). The measurement data were described as mean \pm standard deviation (SD). One-way analysis of variance (ANOVA) with Tukey's post-hoc test was applied for assessing multiple independent groups. Pearson correlation analysis was used to study the relationship between CDKN1B expression and miR-196b-5p. Each experiment was repeated at least three times, and $\mathrm{P}<0.05$ was considered to represent statistical significance.

\section{Results}

\section{Characterization of HemSCs and exosomes}

HemSCs were obtained from proliferating phase IH tissues as described above. In vitro clonal expansion was shown by diluting a single-cell suspension of HemSCs (Figure 1A). Differentiation assay indicated that HemSCs could differentiate into adipocytes, osteocytes (Figure 1B). In addition, qRT-PCR analysis demonstrated that HemSCs highly expressed stem cell marker CD133, as well as CD44, SOX2, LGR5, and NANOG (Figure 1C).

Exosomes were successfully extracted from HemSCs and HUVECs. Transmission electron microscope (TEM) observed specific exosome morphology (Figure 2A).
Nanoparticle size analysis (NTA) showed that the diameter of exosomes was around $100 \mathrm{~nm}$ (Figure 2B). In addition, Western blot confirmed the expression of exosomes' specific surface markers CD63, TSG101, and HSP70 (Figure 2C).

\section{HemSCs-derived exosomes regulated proliferation, apoptosis, cell cycle and angiogenesis of HemECs}

To prove whether HemSCs could exert intercellular effects on HemECs, HemECs were co-cultured with HemSCsexos (HemSCs-exos group), HUVECs-exos (HUVECs-exos group), or cultured independently (HemECs group). First, exosome uptake assay showed that HemECs successfully internalized PKH26-labeled exosomes observed under confocal microscope (Figure $3 A$ ). CCK-8 assay displayed that cell proliferation ability of HemECs in the HemSCsexos group was significantly higher than the HUVECsexos group and the HemECs group after exosomes were taken in for 24-72 h (Figure 3B). In the cell apoptosis assay, cell apoptotic rate was significantly decreased in the HemSCs-exos group than the other two groups (Figure 3C). In addition, there were significantly lower cells arrested in the G0/G1 phase in the HemSCs-exos group compared with the other two groups according to cell cycle assay (Figure 3D). The tube formation ability of the HemSCsexos group was enhanced likewise $(\mathrm{P}<0.05)$ (Figure $3 E)$. Western blot showed that cyclin E, bcl-2 were significantly highly expressed, while the expression of p27, Bax were evidently down-regulated in the HemSCs-exos group $(\mathrm{P}<0.05)$ (Figure $3 F)$. Bax/bcl-2 play a profound role in cell apoptosis, while cyclin E/p27 are closely associated with cell cycle regulation. p27 is encoded by cyclin-dependent kinase inhibitor 1B (CDKN1B). Western blot result was inconsistent with the cell apoptosis assay and cell cycle assay. Notably, HemECs co-cultured with HUVECs-exos for 72 h showed significantly higher proliferation ability than the HemECs group, but lower than the HemSCs-exos group $(\mathrm{P}<0.05)$. A similar trend was observed in cell apoptosis assay. Collectively, these data reflected that the proliferation and angiogenesis abilities of HemECs were enhanced, while cell apoptosis and cell cycle arrest were attenuated in the HemSCs-exos and HemECs co-culture system.

\section{High expression of exosomes derived miR-196b-5p Is detected in HemSCs}

To explore how exosomes regulate intercellular effects between IH cells, this study detected the exosomes-derived 
A

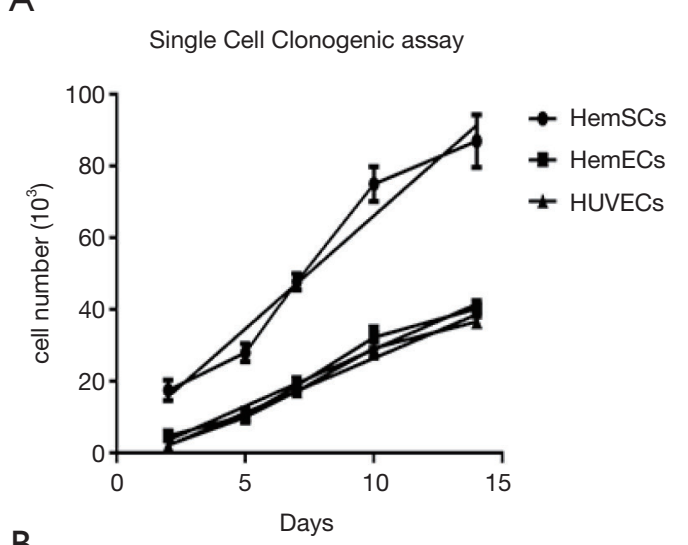

C

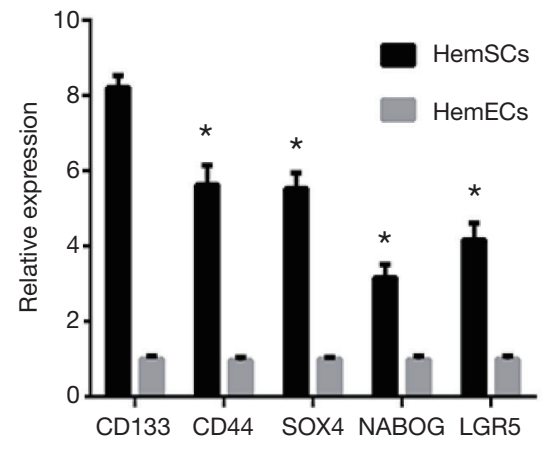

B

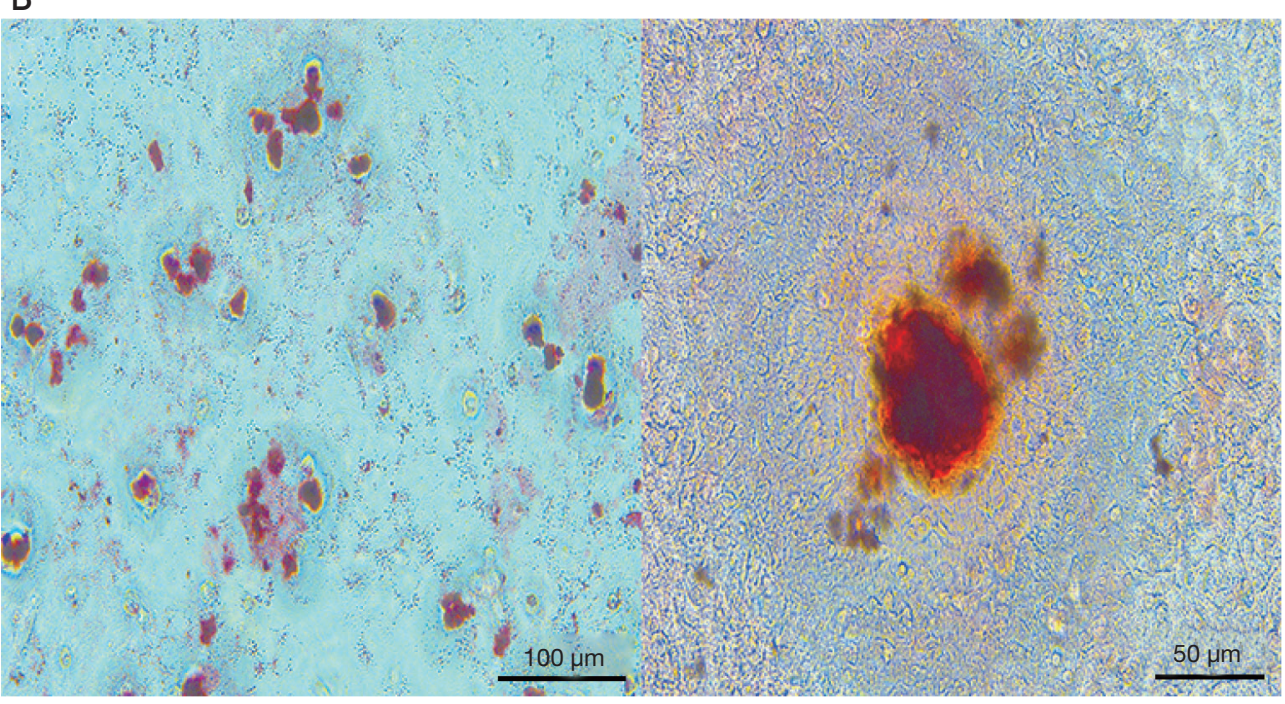

Figure 1 Identification of HemSCs. (A) The clonogenic proliferation ability of HemSCs was analyzed using single cell clonogenic assay. The cell number was calculated on day 2, 5, 7, 10, 14 after single-cell dilution. (B) To examine HemSCs' multiple differentiation ability, HemSCs were cultured in adipogenic, osteogenic media, respectively. Adipogenic ability (left) and osteogenic ability (right) were observed in HemSCs. (C) Stem cell specific markers (CD133, CD44, SOX2, LGR5, NANOG) were examined via qRT-PCR. All experiments were repeated at least 3 times independently. Results were shown as mean \pm standard deviation.

miRNAs in HemSCs by RNA sequencing analysis. miRNA profiles were compared between exosomes from HemSCs and HUVECs. The miRNAs reached a significance level of corrected $\mathrm{P}<0.05$ and had a fold change $>1.5$ were selected and displayed in the volcano plot (Figure $4 A$ ). Ranked by TPM values, the heatmap showed the top 27 differentially expressed miRNAs (Figure 4B). High expression of 6 miRNAs depicted in the heatmap (miR-196b-5p, miR-1301-3p, miR-1307-3p, miR-92b-5p, miR-155-5p, and miR-374a-3p) were further confirmed by qRT-PCR in IH tissue or adjacent normal tissue of 5 patients (Figure 4C).

Both RNA sequencing and qRT-PCR results revealed that miR-196b-5p was one of the most abundantly expressed miRNAs. Moreover, qRT-PCR confirmed that miR-196b-5p exhibited remarkable difference between HemSCs-exos and HUVECs-exos (Figure 4D). Therefore, miR-196b-5p was chosen for further study.

\section{Identification of CDKN1B as a binding target of miR- $196 b-5 p$}

To further demonstrate the downstream target of miR196b-5p, based on the target prediction from RNAhybrid, miRanda, CDKN1B was considered a potential target in 
A
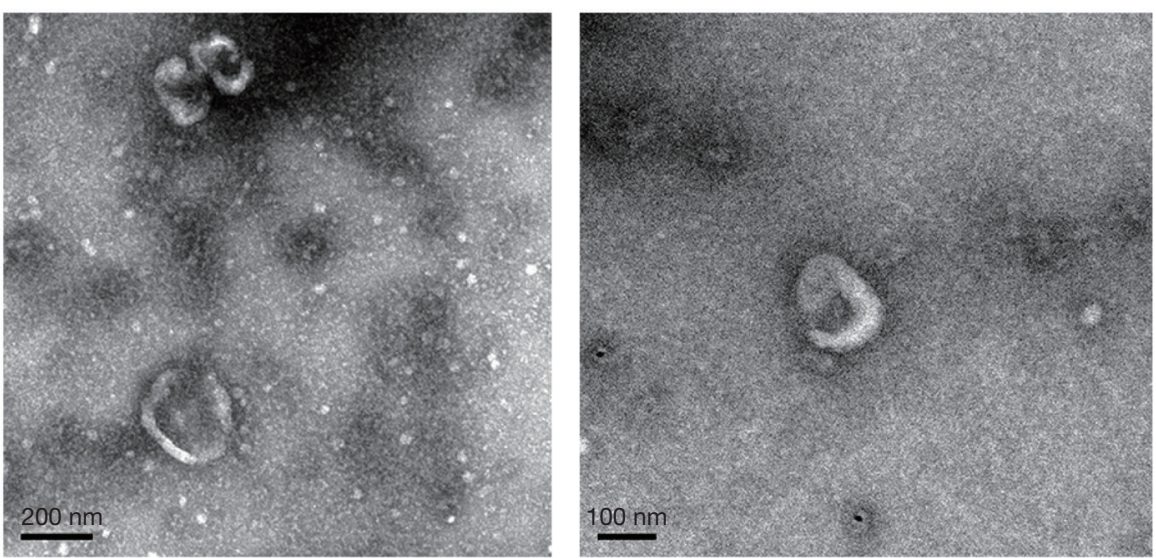

B

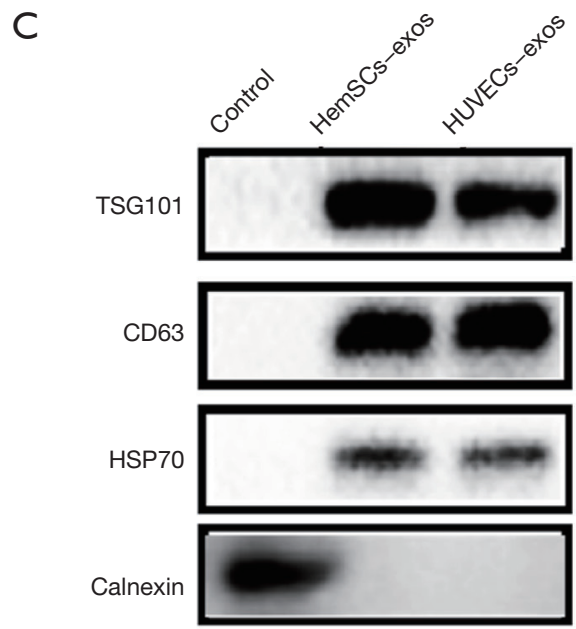

Figure 2 Identification of exosomes derived from HemSCs. (A) Observation of exosome morphology from HemSCs by TEM (scale bar $=200 \mathrm{~nm}$ ) (left) (scale bar =100 nm) (right). (B) Nanoparticle size analysis displayed that the diameter of the extracted exosomes was around $100 \mathrm{~nm}$. (C) Expression of exosome specific surface markers was determined by Western blot. All experiments were repeated at least 3 times independently.

this study. Generally, CDKN1B/p27 inhibited normal cell cycle by interacting with both cyclin and CDK subunits (36), especially cyclin E-cdk2 (37), thus inducing cell cycle arrest by stopping the cell cycle from $\mathrm{G} 1$ to $\mathrm{S}$ phase and regulating cell proliferation. TargetScan database (http://www. targetscan.org) was also used to illustrate the direct binding site between miR-196b-5p and CDKN1B (Figure 5A). In addition, dual-luciferase reporter gene assay was performed to verify the direct binding (Figure 5B). Moreover, a negative correlation was also identified between miR-196b-5p and CDKN1B expression in HemSCs via qRT-PCR (Figure 5C).

To analyze whether miR-196b-5p played an important role in the HemSCs-exos and HemECs co-culture system by binding with CDKN1B, qRT-PCR was performed and confirmed miR-196b-5p and CDKN1B expression level variation (Figure $5 D$ ). These results indicated that miR196b-5p derived from HemSCs-exos generated proliferation, angiogenesis and reduced apoptosis, cell cycle arrest of HemECs via binding with CDKN1B.

\section{Exosomal miR-196b-5p in HemSCs plays a crucial role in the regulation of HemECs}

To further investigate the intercellular role of HemSCsexos-derived miR-196b-5p, HemSCs in the experimental groups were primarily transfected with miR-196b-5p mimic 
A

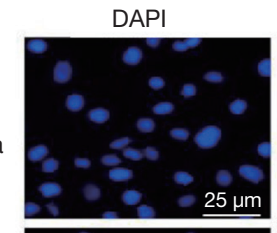

$b$

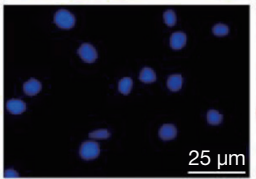

C
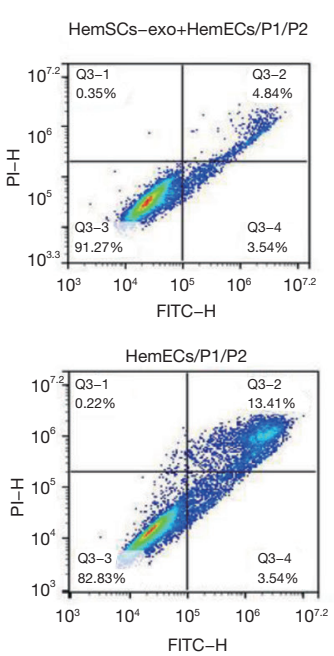

E
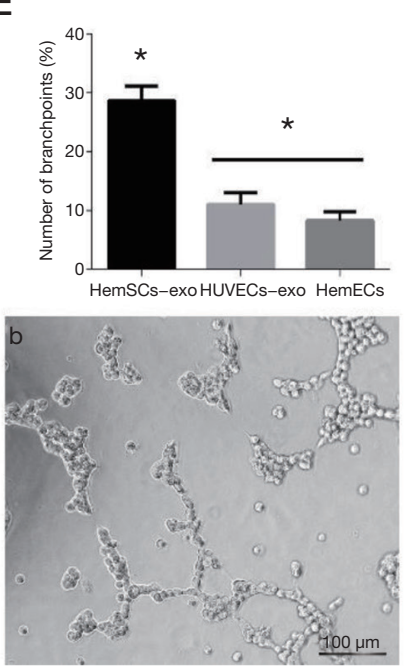

PKH26

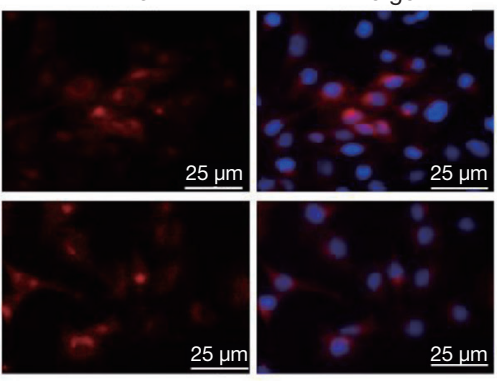

B

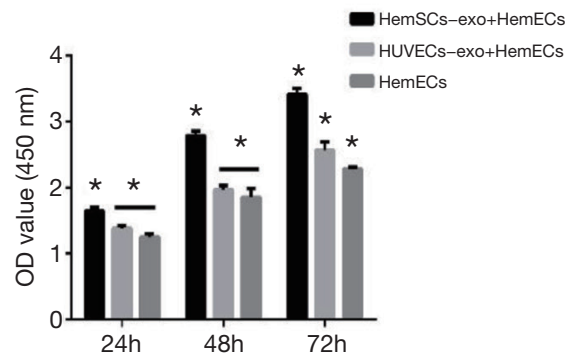

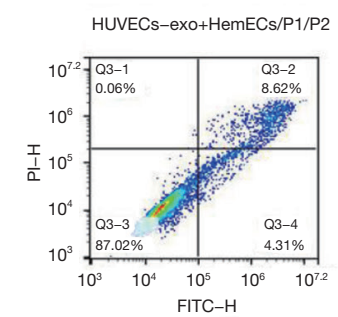

D
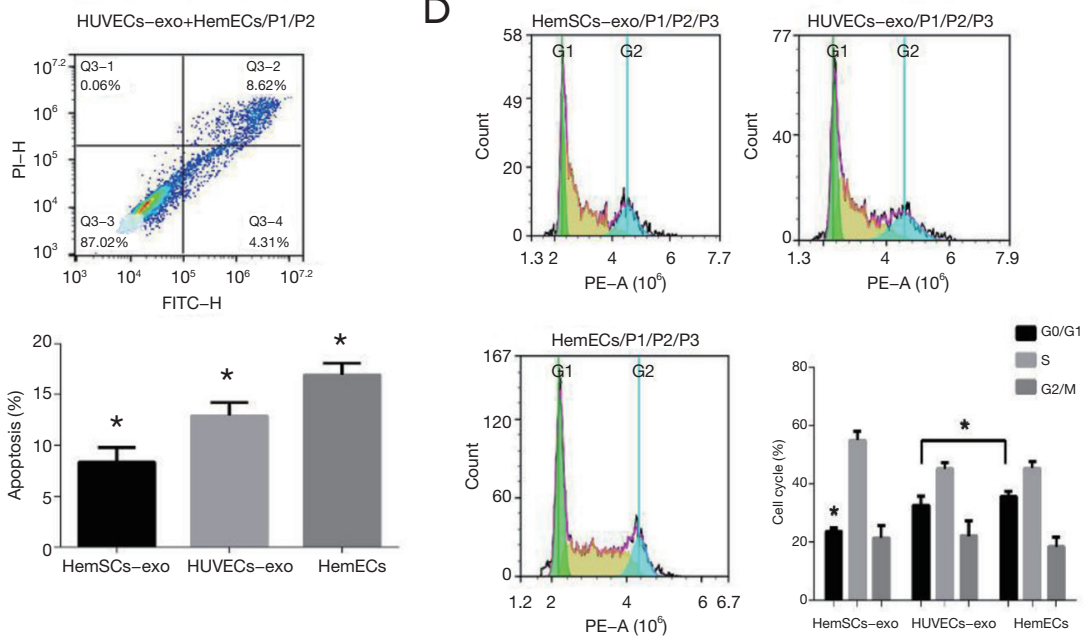

$\mathrm{F}$
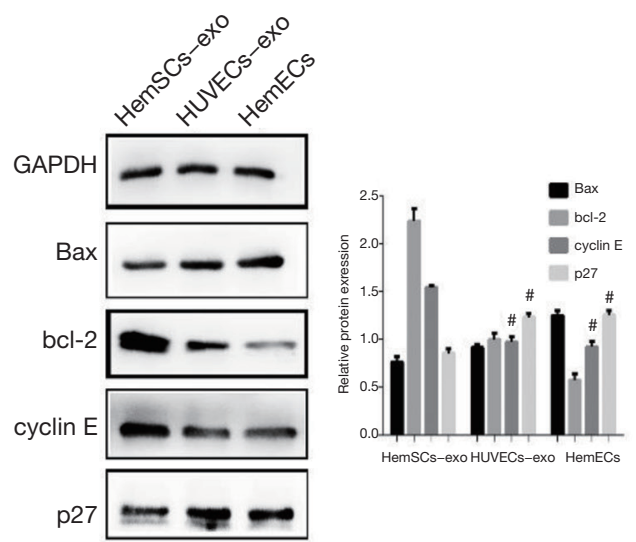

Figure 3 Co-culturing HemECs with HemSCs-exos and HUVECs-exos. (A) Laser confocal microscope $(\times 400)$ confirmed the internalization of HemSCs-Exos and HUVECs-exos in HemECs. (B) CCK-8 assay reflected the viability of HemECs. (C, D) Flow cytometry was utilized to determine the cell apoptosis rate and the cells arrested in the G0/G1 phase. (E) Tube formation assay was conducted to examine the angiogenesis ability of HemECs. a, b, c represented HemSCs-exos group, HUVECs-group, HemECs group, respectively. Scale bar $=100 \mu \mathrm{m}$. (F) Western blot showed the expression levels of cyclin E, bcl-2, p27, Bax. All experiments were repeated at least 3 times independently. Results are shown as mean \pm standard deviation. ${ }^{*}$ represented that there was no significant difference between groups. ${ }^{*} \mathrm{P}<0.05$. 
A

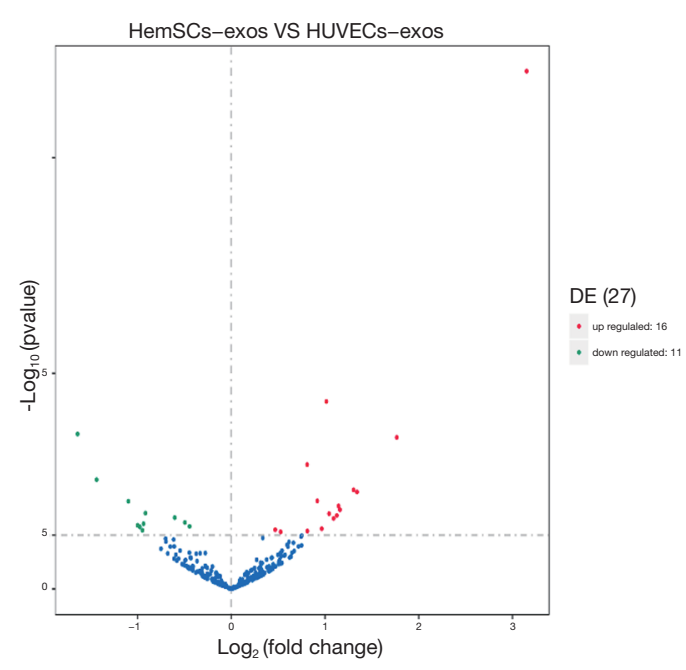

C

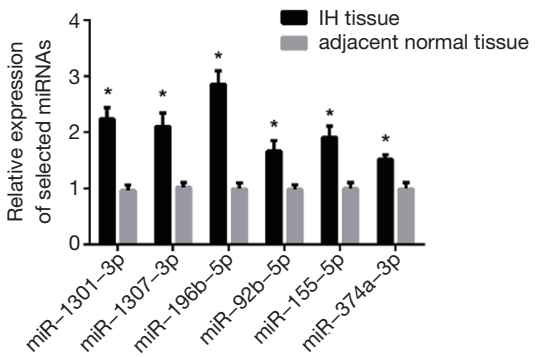

B

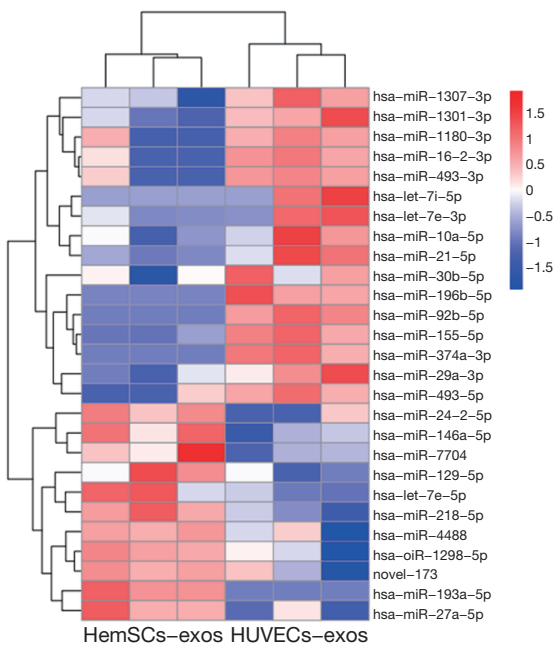

D

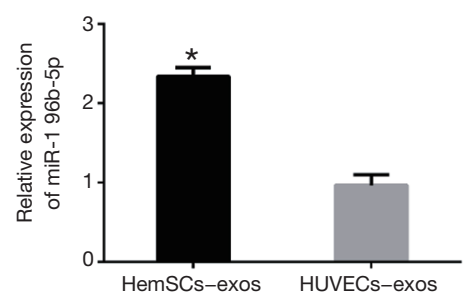

Figure 4 miR-196b-5p was highly expressed in HemSCs-exos and IH tissues. (A) The volcano plot depicted the differentially expressed miRNAs in HemSCs-exos and HUVECs-exos. The up-regulated and down-regulated miRNAs in HemSCs-exos reaching a significance level of corrected $\mathrm{P}<0.05$ and a fold change $>1.5$ were selected. (B) Differentially expressed miRNAs ranked by TPM values were shown in the heatmap. (C) qRT-PCR detected that the expression level of miRNAs in IH tissues. Among 6 miRNAs, miR-196b-5p was the most abundantly expressed miRNA. (D) qRT-PCR showed the expression level of miR-196b-5p in HemSCs-exos and HUVECs-exos. All experiments were repeated at least 3 times independently. Results are shown as mean \pm standard deviation. ${ }^{*} \mathrm{P}<0.05$.

(miR-196b-5p mimic group) or inhibitor (miR-196b-5p inhibitor group) to alter endogenous miR-196b-5p level, while HemSCs in the control groups were transfected with NC mimic (NC mimic group) or NC inhibitor (NC inhibitor group). qRT-PCR showed that expression of miR$196 \mathrm{~b}-5 \mathrm{p}$ was up-regulated or down-regulated in exosomes derived from miR-196b-5p mimic or miR-196b-5p inhibitor transfected HemSCs (Figure 6A). After co-culturing HemECs with exosomes derived from HemSCs for $48 \mathrm{~h}$, the proliferating rate in the miR-196b-5p mimic group was significantly increased compared with the miR-196b-5p inhibitor group (Figure 6B). Conversely, apoptosis rate in the miR-196b-5p inhibitor group was dramatically higher than the miR-196b-5p mimic group $(\mathrm{P}<0.05)$ (Figure 6C). Cell cycle assay showed significantly more cells in the G0/
G1 phase in the miR-196b-5p inhibitor group (Figure 6D). In the miR-196b-5p mimic group, tube formation assay indicated the enhancement of angiogenesis ability, whereas the tube formation was evidently decreased in the miR196b-5p inhibitor group $(\mathrm{P}<0.05)$ (Figure 6E). qRT-PCR manifested that miRNA-196b-5p were overtly up-regulated and CDKN1B were down-regulated in the miR-196b-5p mimic group, whereas the miR-196b-5p inhibitor group produced a converse result $(\mathrm{P}<0.05)$ (Figure 6F). Western blot revealed that the expression of cyclin $\mathrm{E}$, bcl-2 were significantly increased whereas p27, Bax were decreased in the miR-196b-5p mimic group $(\mathrm{P}<0.05)$ (Figure 6G). Taken together, these data confirmed that exosomes derived miR-196b-5p from HemSCs could facilitate intercellular communication between HemSCs and HemECs. 
A

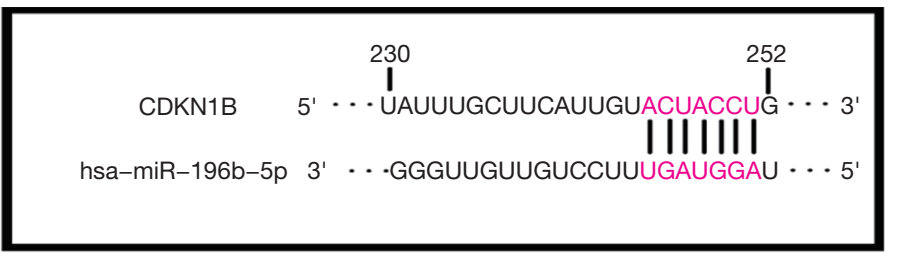

B

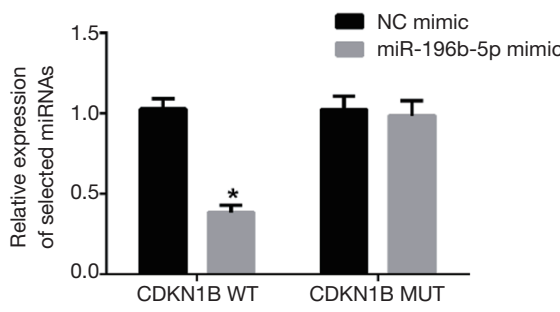

C

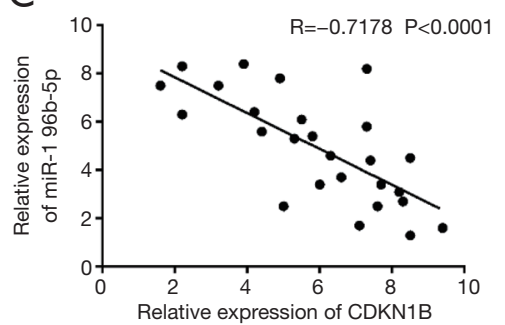

D
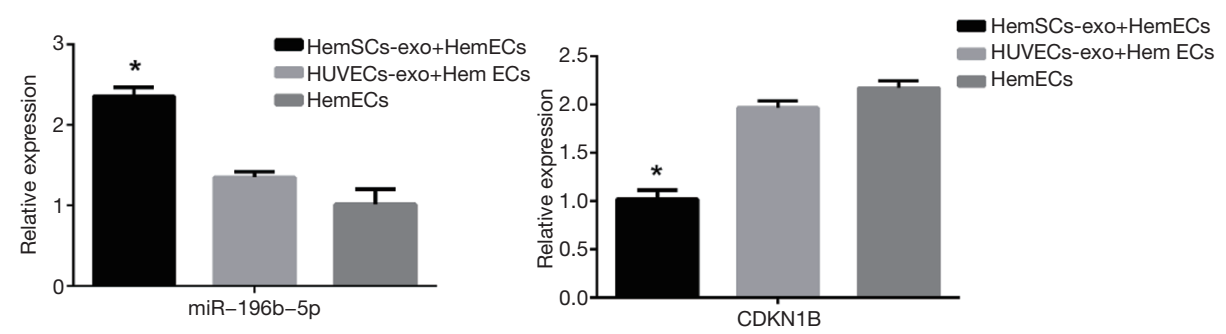

Figure 5 CDKN1B was identified as a binding target of miR-196b-5p. (A) TargetScan database was used to predict the binding sites of miR196b-5p on CDKN1B. (B) The binding relationship between miR-196b-5p and CDKN1B was confirmed by dual-luciferase reporter gene assay. (C) Correlation analysis revealed that there was a negative correlation between miR-196b-5p and CDKN1B in HemSCs. (D) qRTPCR confirmed the expression level of miR-196b-5p and CDKN1B. All experiments were repeated at least 3 times independently. Results are shown as mean \pm standard deviation. ${ }^{*} \mathrm{P}<0.05$.

\section{MiR-196b-5p functions in HemECs via down-regulating CDKN1B}

In the HemSCs-exos group, qRT-PCR and Western blot displayed significantly lower expression of CDKN1B at both transcription and protein level compared with the other the two groups as described above. To verify whether miR196b-5p inhibited CDKN1B expression to alter HemECs' abilities, HemECs were transfected with oe-CDKN1B (oeCDKN1B group) or oe-NC (oe-NC group) and co-cultured with exosomes from HemSCs transfected with miR-196b$5 \mathrm{p}$ mimics for $48 \mathrm{~h}$. First, qRT-PCR was performed to detect the transfection efficiency in HemECs (Figure $7 A$ ). In CCK-8 assay and tube formation assay, the oe-CDKN1B group had significantly lower proliferation and angiogenesis ability in contrast to the oe-NC group (Figure $7 B, C$ ). Flow cytometry illustrated significantly lower apoptosis and cell cycle repression rate in the oe-NC group (Figure $7 D, E$ ). The transcription level of CDKN1B in HemECs was detected by qRT-PCR (Figure 7F). Similarly, cyclin E, Bcl-2 were expressed significantly higher in the oe-NC group, whereas p27, Bax were expressed significantly higher in the oeCDKN1B group (Figure $7 G$ ). Collectively, CDKN1B was identified as a downstream binding target of miR-196b-5p, capable of regulating cell cycle, apoptosis, proliferation, and angiogenesis abilities of HemECs.

\section{Discussion}

$\mathrm{IH}$ is a benign vascular neoplasm and the most common soft tissue tumor of infancy. However, the pathogenesis of IH remains unclear. Hence, it's essential to further investigate the underlying mechanism, and explore novel biomarkers for targeted therapy. In this study, exosomes from HemSCs were proved to play an indispensable role in IH progression. HemSCs could regulate HemECs' proliferation, cell cycle, 


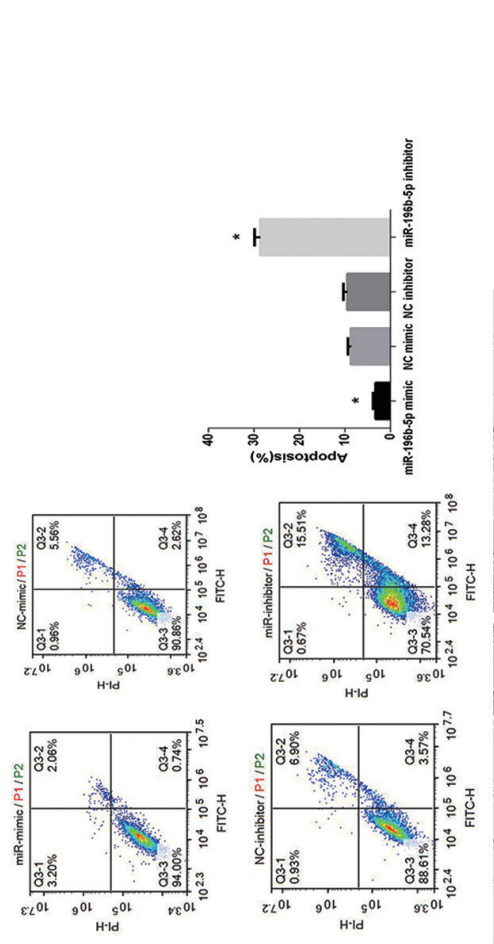

U

$\infty$

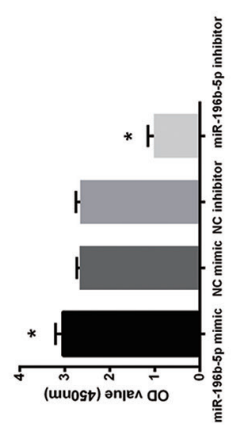

ш

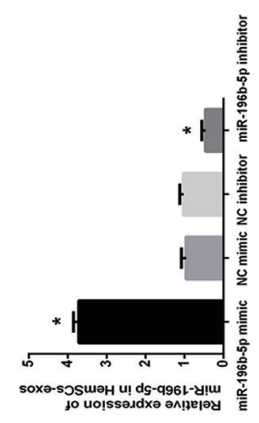

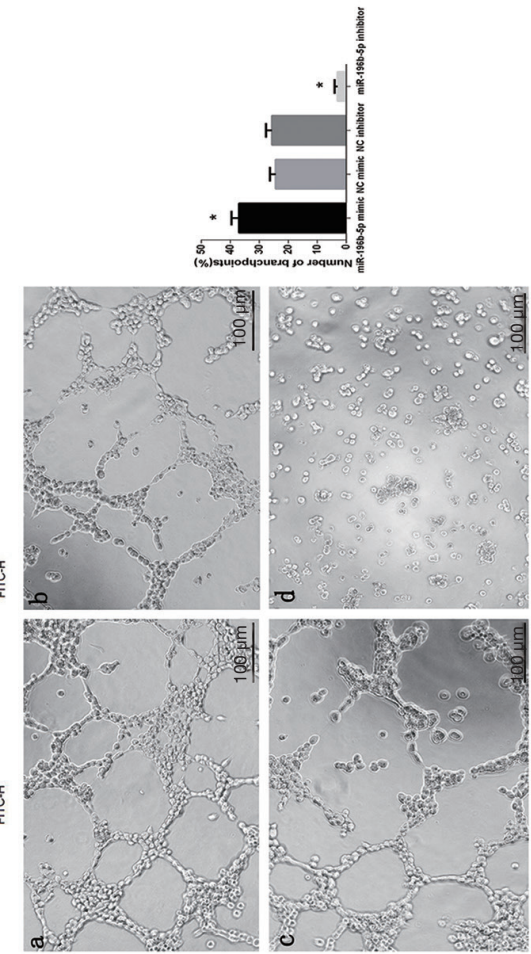

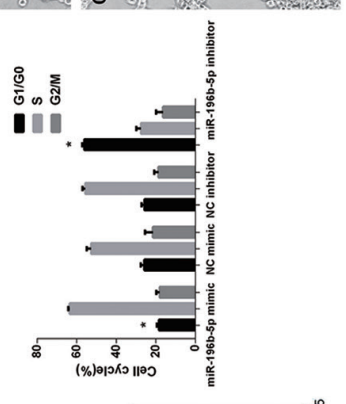

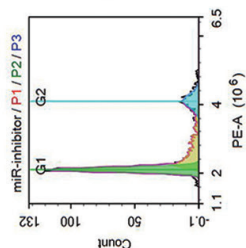
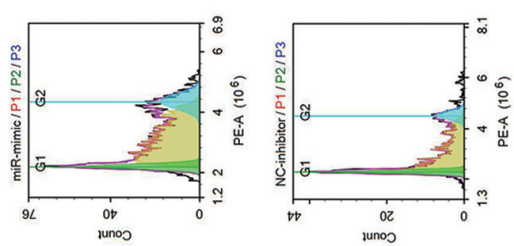

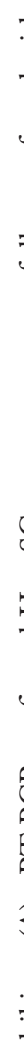

v
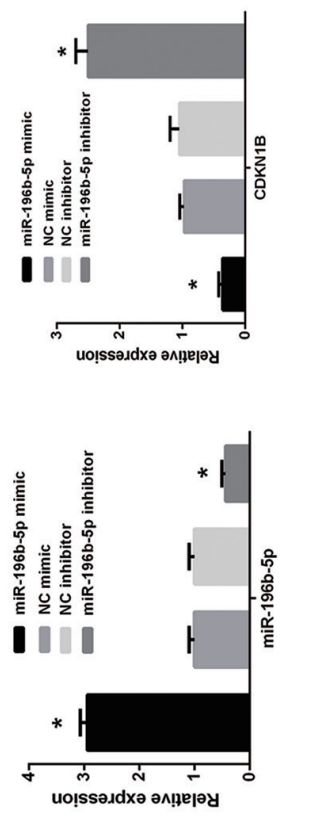

レ

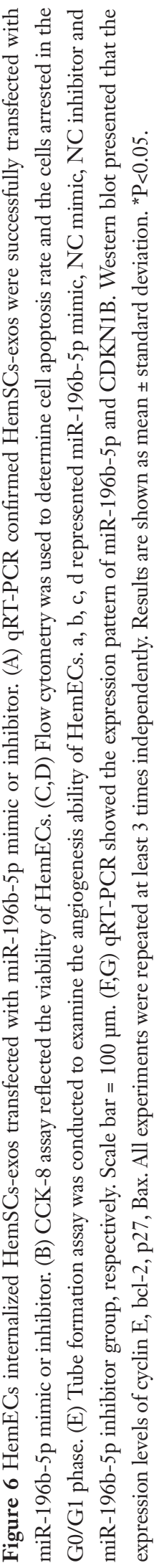


A
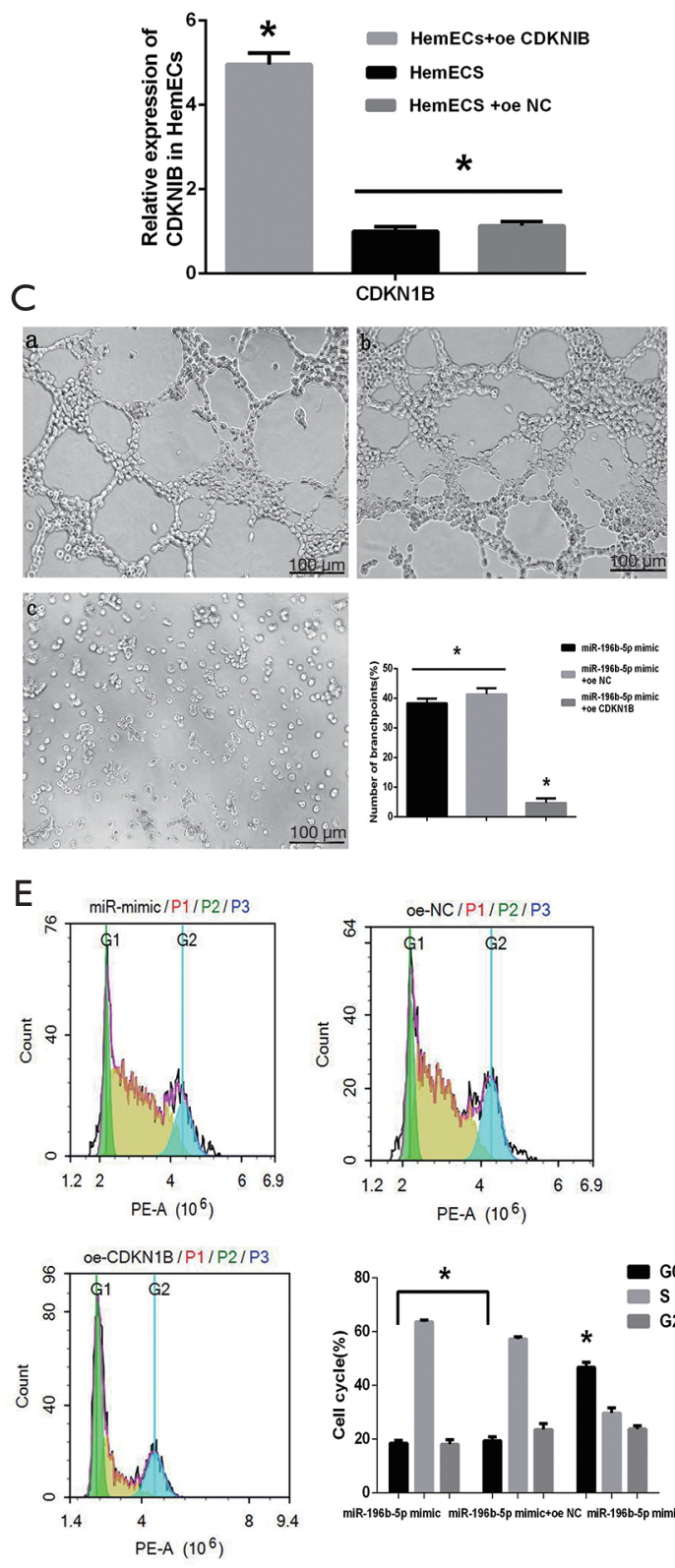

B

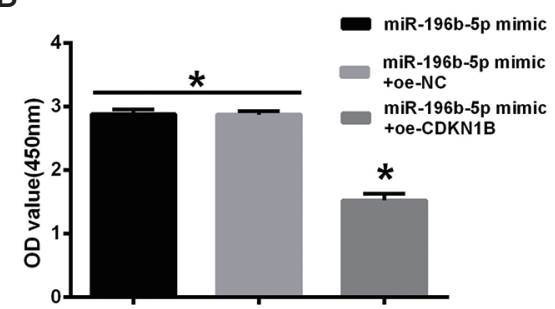

D
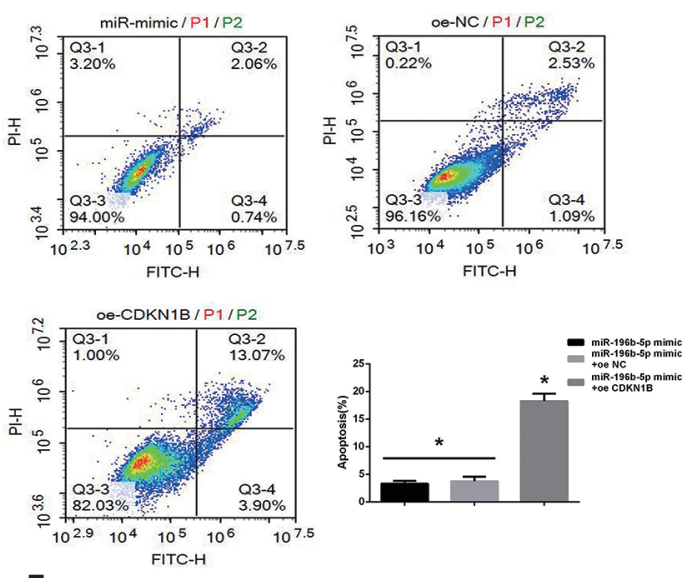

$\mathrm{F}$
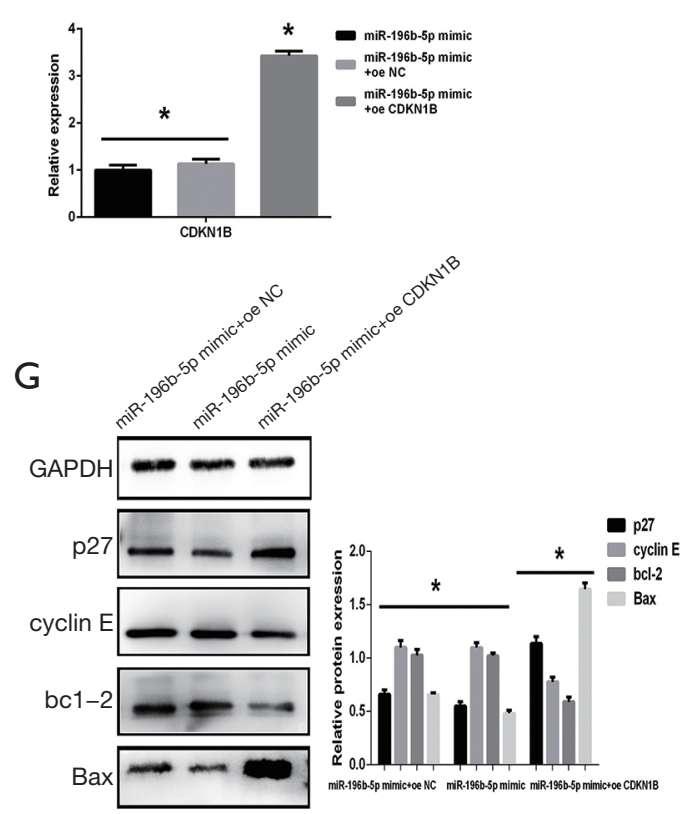

Figure 7 HemECs were transfected with oe-CDKNIB. (A) qRT-PCR confirmed that HemECs were successfully transfected with oeCDKNIB. (B) CCK-8 assay reflected cell viability. (C) Tube formation assay was conducted to examine the angiogenesis ability of HemECs. a, b, c represented miR-196b-5p mimic, oe-NC and oe-CDKN1B group, respectively. Scale bar $=100 \mu \mathrm{m}$. (D,E) Flow cytometry was used to determine cell apoptosis rate and the cells arrested in the G0/G1 phase. (F,G) qRT-PCR showed the expression level of CDKN1B. Western blot presented the expression levels of cyclin E, bcl-2, p27, Bax. All experiments were repeated at least 3 times independently. Results are shown as mean \pm standard deviation. ${ }^{*} \mathrm{P}<0.05$. 
apoptosis and angiogenesis via exosomes. miR-196b-5p from HemSCs-exos was a pivotal factor in this process because it directly bound with CDKN1B and regulate various abilities of HemECs.

Increasing evidence showed that tumor cells-derived exosomes facilitated intercellular communications and promoted tumor progression via TME. TME is a complicated biosystem comprised blood vessels, nerve fibers, immune cells, other nonmalignant cells, and EVs (38). Numerous studies have demonstrated that EVs, especially exosomes, in TME could promote crosstalk between distinct cells. Pang et al. (39) revealed that oral squamous cell carcinoma (OSCC) cells derived-exosomes stimulated M2like macrophage polarization in OSCC microenvironment, resulting in malignant progression. Moreover, it was reported that cancer-associated fibroblasts (CAFs) endowed chemotherapy resistance to head and neck cancer cells via autocrine exosomes in TME (40). To investigate the interaction effects between HemSCs and HemECs in IH microenvironment, HemECs were cultured with HemSCs' exosomes. We surprisingly found that the proliferation and angiogenesis ability of HemECs were significantly increased in comparison with the HUVECs-exos group and the HemECs group. In addition, apoptosis rate and cell percentages at G0/G1 phases were reduced in the HemSCs-exos group, too. Few studies have focused on the relationship between exosomes and pathogenesis of IH. According to our knowledge, this study might be the first to explore the function of exosomes between hemangioma cells. HUVECs are common cell lines used as the negative control in many IH studies. HUVECs do not have the stem cell property, which HemSCs are endowed with. Notably, in the present study, HUVECs-exos also exerted positive effect on HemECs under certain conditions, though the effect was significantly lower than that in the HemSCs-exos group. The anti-apoptotic effect of HUVECs-exos was in consistent with previous results. Evidence has shown that HUVECsexos could induce anti-apoptotic influence on neural cells $(41,42)$. Inhibition of miR-21-3p's binding target ATG12 regulated the apoptotic pathway by suppressing autophagy or inactivating Bcl-2 family members (42).

It has been frequently reported that miR-196b-5p was involved in tumor progression. Abundantly expressed miR196b-5p was observed in several tumors, such as colorectal cancer (43), lung cancer $(44,45)$, and liver cancer $(46)$. miR196b-5p could directly target the tumor suppressor, thus accelerating tumor cell proliferation, migration, and cell cycle $(43,44)$. In addition, it was reported that miR-196b-
$5 \mathrm{p}$ was remarkably enriched in cells-derived exosomes, generating crosstalk between different types of cell $(47,48)$. This study found that miR-196b-5p was a potential highly expressed miRNA in HemSCs-exos by RNA sequencing analysis. According to these results, we speculated that miR-196b-5p might be essential for the interaction between HemSCs and HemECs. To further validate this presumption, HemSCs were transfected with miR-196b$5 p$ mimic or miR-196b-5p inhibitor. In the miR-196b-5p mimic group, the proliferation and angiogenesis ability of HemECs were evidently increased whereas apoptosis rate and cell percentages at G0/G1 phases were significantly decreased. The miR-196b-5p inhibitor group displayed an opposite result. The impact of miRNAs in IH has not been fully discovered yet. Zeng et al. (49) suggested that miR-501 had an oncogenic role in the pathogenesis of IH. MiR-501 promoted IH cell line proliferation, migration, and invasion by targeting homeobox D10 (HOXD10). Li et al. (50) identified that the downregulation of miR-382 by propranolol prohibited the progression of IH through the PTEN-mediated AKT/mTOR pathway. Few studies have connected IH with miR-196. The specific function of IH related exosomal miR-196b-5p remains unclear.

Next, the current study aimed to find the direct target of miR-196b-5p. According to several databases, CDKN1B was considered the potential target gene. Generally, Cyclindependent kinase inhibitor 1B (CDKN1B) encodes p27, which is a well-known tumor-inhibiting factor from the Kinase Inhibitory Protein (Kip) family (51,52). Because of the ability to regulate the cell cycle and growth, CDKN1B is known as a tumor suppressor (53). Previous studies documented the participation of p27 in IH progression. Pan et al. (54) demonstrated that propranolol disrupted the cell cycle of HemECs through positively altering p27 expression, thus inducing IH regression. In this study, to verify that CDKN1B was the pivotal target of miR-196b$5 \mathrm{p}$, orchestrating the interaction between HemSCs and HemECs, HemECs were transfected with oe-CDKN1B or oe-NC and co-cultured with exosomes from miR-196b-5p over-expressed HemSCs. In the oe-CDKN1B group, overexpressed CDKN1B compensated miR-196b-5p's inhibitory effects on CDKN1B, hence HemECs' proliferation and angiogenesis ability were significantly attenuated. In contrast, apoptosis assay and cell cycle assay exhibited a higher apoptosis rate and cell percentages at G0/G1 phases. The negative effect of CDKN1B on cell apoptosis and cell cycle was confirmed by $\mathrm{p} 27$, cycle E, bcl-2, and Bax expression levels. From these results, the present study 
reflected that miR-196b-5p and CDKN1B played a crucial role in HemSCs-exos' regulation of HemECs.

Apart from cell cycle repression ability, previous studies also found that CDKN1B could induce cell apoptosis $(55,56)$ independent of its CDK inhibitory action. Several studies reported that CDKN1B induced cell apoptosis via suppressing hsp27 and hsp70 transcriptional expression (57), or it might be related to $\mathrm{PI} 3 \mathrm{~K} / \mathrm{Akt} / \mathrm{mTOR}$ signaling pathway (58). It must be noted that there are controversies over CDKN1B's effect on cell apoptosis. Chen et al. (51) demonstrated that oxyhemoglobin reduced the level of CDKN1B in neuronal cells, inducing apoptosis and inflammation. CDKN1B overexpression would reverse the negative effect of oxyhemoglobin. Liang et al. (37) reported that p27 is downstream of LKB1-AMPK energy-sensing pathway. p27 depletion led to cell apoptosis, while stabilized p27 wound permit cells survived. However, this study showed that p27 apparently induced HemECs apoptosis. This apoptosis induction tendency is identical to previous studies (55-58). Moreover, this study found that miR-196b-5p facilitated the tube formation via down-regulating CDKN1B. According to relevant previous studies, we speculated that angiogenesis suppression was probably due to CDKN1B's negative effect on endothelial cell proliferation and migration (59-62). This study displayed a similar result as well. miR-196b-5p exerted the anti-angiogenesis influence on HemECs. This might be due to the decline of CDKN1B, inducing the suppression of HemECs proliferation ability, other than classical angiogenesis signaling pathway regulation.

Briefly, the current study discovered the role of exosomes in $\mathrm{IH}$ progression. HemSCs-exos derived miR-196b-5p could exert intercellular effects on HemECs via directly targeting CDKN1B. This is the first report on the case of exosomes in IH. However, whether HemECs could regulate HemSCs conversely, via exosomes or other ways, needs to be investigated in the future. Moreover, nude mouse models ought to be constructed in further studies to evaluate the effect of miR-196b-5p and CDKN1B on tumor development in vivo.

\section{Conclusions}

This study found a negative correlation between miR-196b$5 \mathrm{p}$ and CDKN1B expression in IH microenvironment and confirmed that HemSCs-exos-derived miR-196b-5p was crucial for exerting intercellular effect on HemECs. In addition, CDKN1B was confirmed as the direct target of miR-196b-5p. miR-196b-5p from HemSCs-exos regulated proliferation, cell cycle, angiogenesis, and apoptosis of HemECs by directly binding with CDKN1B. These findings can probably provide a novel predictor and potential therapeutic target for IH diagnosis and treatment.

\section{Acknowledgments}

Funding: This study was financially supported by the grants of National Natural Science Foundation of China [81771087, 81901021].

\section{Footnote}

Reporting Checklist: The authors have completed the MDAR checklist. Available at http://dx.doi.org/10.21037/atm-206456

Data Sharing Statement: Available at http://dx.doi. org/10.21037/atm-20-6456

Conflicts of Interest: All authors have completed the ICMJE uniform disclosure form (available at http://dx.doi. org/10.21037/atm-20-6456). The authors have no conflicts of interest to declare.

Etbical Statement: The authors are accountable for all aspects of the work in ensuring that questions related to the accuracy or integrity of any part of the work are appropriately investigated and resolved. The study was conducted in accordance with the Declaration of Helsinki (as revised in 2013). The study was approved by the Institutional Ethics Committee of Shanghai Ninth People's Hospital (SH9H2019-TK168-1) and informed consent was taken from all individual participants.

Open Access Statement: This is an Open Access article distributed in accordance with the Creative Commons AttributionNonCommercial-NoDerivs 4.0 International License (CC BYNC-ND 4.0), which permits the non-commercial replication and distribution of the article with the strict proviso that no changes or edits are made and the original work is properly cited (including links to both the formal publication through the relevant DOI and the license). See: https://creativecommons. org/licenses/by-nc-nd/4.0/.

\section{References}

1. Solman L, Glover M, Beattie PE, et al. Oral propranolol 
in the treatment of proliferating infantile haemangiomas: British Society for Paediatric Dermatology consensus guidelines. Br J Dermatol 2018;179:582-9.

2. Léauté-Labrèze C, Hoeger $\mathrm{P}$, Mazereeuw-Hautier J, et al. A randomized, controlled trial of oral propranolol in infantile hemangioma. N Engl J Med 2015;372:735-46.

3. Liu C, Zhao Z, Ji Z, et al. MiR-187-3p Enhances Propranolol Sensitivity of Hemangioma Stem Cells. Cell Struct Funct 2019;44:41-50.

4. Khan ZA, Boscolo E, Picard A, et al. Multipotential stem cells recapitulate human infantile hemangioma in immunodeficient mice. J Clin Invest 2008;118:2592-9.

5. Harbi S, Wang R, Gregory M, et al. Infantile Hemangioma Originates from A Dysregulated but Not Fully Transformed Multipotent Stem Cell. Sci Rep 2016;6:35811.

6. Kleiman A, Keats EC, Chan NG, et al. Evolution of hemangioma endothelium. Exp Mol Pathol 2012;93:264-72.

7. Edwards AK, Glithero K, Grzesik P, et al. NOTCH3 regulates stem-to-mural cell differentiation in infantile hemangioma. JCI Insight 2017;2:e93764.

8. Greenberger S, Boscolo E, Adini I, et al. Corticosteroid suppression of VEGF-A in infantile hemangioma-derived stem cells. N Engl J Med 2010;362:1005-13.

9. Jinnin M, Medici D, Park L, et al. Suppressed NFATdependent VEGFR1 expression and constitutive VEGFR2 signaling in infantile hemangioma. Nat Med 2008;14:1236-46.

10. Smadja DM, Mulliken JB, Bischoff J. E-selectin mediates stem cell adhesion and formation of blood vessels in a murine model of infantile hemangioma. Am J Pathol 2012;181:2239-47.

11. Jeppesen DK, Fenix AM, Franklin JL, et al. Reassessment of Exosome Composition. Cell 2019;177:428-45.e18.

12. Crewe C, Joffin N, Rutkowski JM, et al. An Endothelial-toAdipocyte Extracellular Vesicle Axis Governed by Metabolic State. Cell 2018;175:695-708.e13.

13. Wortzel I, Dror S, Kenific CM, et al. Exosome-Mediated Metastasis: Communication from a Distance. Dev Cell 2019;49:347-60.

14. Shimoda M, Khokha R. Metalloproteinases in extracellular vesicles. Biochim Biophys Acta Mol Cell Res 2017;1864:1989-2000.

15. Xu R, Greening DW, Zhu HJ, et al. Extracellular vesicle isolation and characterization: toward clinical application. J Clin Invest 2016;126:1152-62.

16. Lakhter AJ, Pratt RE, Moore RE, et al. Beta cell extracellular vesicle miR-21-5p cargo is increased in response to inflammatory cytokines and serves as a biomarker of type 1 diabetes. Diabetologia 2018;61:1124-34.

17. Letsiou E, Bauer N. Endothelial Extracellular Vesicles in Pulmonary Function and Disease. Curr Top Membr 2018;82:197-256.

18. Zhan R, Leng X, Liu X, et al. Heat shock protein 70 is secreted from endothelial cells by a non-classical pathway involving exosomes. Biochem Biophys Res Commun 2009;387:229-33.

19. Sun J, Zhang Z, Ma T, et al. Endothelial progenitor cellderived exosomes, loaded with miR-126, promoted deep vein thrombosis resolution and recanalization. Stem Cell Res Ther 2018;9:223.

20. Ke X, Yang D, Liang J, et al. Human Endothelial Progenitor Cell-Derived Exosomes Increase Proliferation and Angiogenesis in Cardiac Fibroblasts by Promoting the Mesenchymal-Endothelial Transition and Reducing High Mobility Group Box 1 Protein B1 Expression. DNA Cell Biol 2017;36:1018-28.

21. Du J, Liang Y, Li J, et al. Gastric Cancer Cell-Derived Exosomal microRNA-23a Promotes Angiogenesis by Targeting PTEN. Front Oncol 2020;10:326.

22. Huang L, Nakayama H, Klagsbrun M, et al. Glucose transporter 1-positive endothelial cells in infantile hemangioma exhibit features of facultative stem cells. Stem Cells 2015;33:133-45.

23. Herter EK, Xu Landén N. Non-Coding RNAs: New Players in Skin Wound Healing. Adv Wound Care (New Rochelle) 2017;6:93-107.

24. Xu Y, Wu D, Jiang Z, et al. MiR-616-3p modulates cell proliferation and migration through targeting tissue factor pathway inhibitor 2 in preeclampsia. Cell Prolif 2018;51: e12490.

25. Wu L, Song WY, Xie Y, et al. miR-181a-5p suppresses invasion and migration of HTR-8/SVneo cells by directly targeting IGF2BP2. Cell Death Dis 2018;9:16.

26. Yang H, Zhang H, Ge S, et al. Exosome-Derived miR130a Activates Angiogenesis in Gastric Cancer by Targeting C-MYB in Vascular Endothelial Cells. Mol Ther 2018;26:2466-75.

27. Tkach M, Théry C. Communication by Extracellular Vesicles: Where We Are and Where We Need to Go. Cell 2016;164:1226-32.

28. Yang F, Ning Z, Ma L, et al. Exosomal miRNAs and miRNA dysregulation in cancer-associated fibroblasts. Mol Cancer 2017;16:148.

29. Mathiyalagan P, Liang Y, Kim D, et al. Angiogenic 
Mechanisms of Human CD34+ Stem Cell Exosomes in the Repair of Ischemic Hindlimb. Circ Res 2017;120:1466-76.

30. Dellett M, Brown ED, Guduric-Fuchs J, et al. MicroRNAcontaining extracellular vesicles released from endothelial colony-forming cells modulate angiogenesis during ischaemic retinopathy. J Cell Mol Med 2017;21:3405-19.

31. Hu H, Wang B, Jiang C, et al. Endothelial progenitor cell-derived exosomes facilitate vascular endothelial cell repair through shuttling miR-21-5p to modulate Thrombospondin-1 expression. Clin Sci (Lond) 2019; 133:1629-44.

32. Khan ZA, Melero-Martin JM, Wu X, et al. Endothelial progenitor cells from infantile hemangioma and umbilical cord blood display unique cellular responses to endostatin. Blood 2006;108:915-21.

33. Wang Q, Liu J, Guo T, et al. Epidermal Growth Factor Reverses the Inhibitory Effects of the Bisphosphonate, Zoledronic Acid, on Human Oral Keratinocytes and Human Vascular Endothelial Cells In Vitro via the Epidermal Growth Factor Receptor (EGFR)/Akt/ Phosphoinositide 3-Kinase (PI3K) Signaling Pathway. Med Sci Monit 2019;25:700-10.

34. Liu H, Liu Y, Sun P, et al. Colorectal cancer-derived exosomal miR-106b-3p promotes metastasis by down-regulating DLC-1 expression. Clin Sci (Lond) 2020;134:419-34.

35. Chen Y, Song Y, Huang J, et al. Increased Circulating Exosomal miRNA-223 Is Associated with Acute Ischemic Stroke. Front Neurol 2017;8:57.

36. Chu IM, Hengst L, Slingerland JM. The Cdk inhibitor p27 in human cancer: prognostic potential and relevance to anticancer therapy. Nat Rev Cancer 2008;8:253-67.

37. Liang J, Shao SH, Xu ZX, et al. The energy sensing LKB1AMPK pathway regulates p27(kip1) phosphorylation mediating the decision to enter autophagy or apoptosis. Nat Cell Biol 2007;9:218-24.

38. Kok VC, Yu CC. Cancer-Derived Exosomes: Their Role in Cancer Biology and Biomarker Development. Int J Nanomedicine 2020;15:8019-36.

39. Pang X, Wang SS, Zhang M, et al. OSCC cell-secreted exosomal CMTM6 induced M2-like macrophages polarization via ERK1/2 signaling pathway. Cancer Immunol Immunother 2020. [Epub ahead of print]. doi: 10.1007/s00262-020-02741-2.

40. Qin X, Guo H, Wang X, et al. Exosomal miR-196a derived from cancer-associated fibroblasts confers cisplatin resistance in head and neck cancer through targeting CDKN1B and ING5. Genome Biol 2019;20:12.
41. Yu Y, Zhou H, Xiong Y, et al. Exosomal miR-199a-5p derived from endothelial cells attenuates apoptosis and inflammation in neural cells by inhibiting endoplasmic reticulum stress. Brain Res 2020;1726:146515.

42. Jiang Y, Xie H, Tu W, et al. Exosomes secreted by HUVECs attenuate hypoxia/reoxygenation-induced apoptosis in neural cells by suppressing miR-21-3p. Am J Transl Res 2018;10:3529-41.

43. Xin H, Wang C, Chi Y, et al. MicroRNA-196b-5p promotes malignant progression of colorectal cancer by targeting ING5. Cancer Cell Int 2020;20:119.

44. Liang G, Meng W, Huang X, et al. miR-196b-5p-mediated downregulation of TSPAN12 and GATA6 promotes tumor progression in non-small cell lung cancer. Proc Natl Acad Sci U S A 2020;117:4347-57.

45. Mao X, Zhou X, Liu J, et al. Up-regulated Linc00472 suppresses development of lung cancer cell via inhibition of MiR-196b-5p. Biosci Biotechnol Biochem 2019:1-13. doi: 10.1080/09168451.2019.1694404.

46. Zhang L, Luo B, Dang YW, et al. Clinical Significance of microRNA-196b-5p in Hepatocellular Carcinoma and its Potential Molecular Mechanism J Cancer. 2019;10:5355-70.

47. Hu R, Li X, Peng C, et al. miR-196b-5p-enriched extracellular vesicles from tubular epithelial cells mediated aldosterone-induced renal fibrosis in mice with diabetes. BMJ Open Diabetes Res Care 2020;8: e001101.

48. Marinaro F, Gómez-Serrano M, Jorge I, et al. Unraveling the Molecular Signature of Extracellular Vesicles from Endometrial-Derived Mesenchymal Stem Cells: Potential Modulatory Effects and Therapeutic Applications. Front Bioeng Biotechnol 2019;7:431.

49. Zeng Z, Liu S, Cai J, et al. miR-501 promotes hemangioma progression by targeting HOXD10. Am J Transl Res 2019;11:2439-46.

50. Li D, Li P, Guo Z, et al. Downregulation of miR-382 by propranolol inhibits the progression of infantile hemangioma via the PTEN-mediated AKT/mTOR pathway. Int J Mol Med 2017;39:757-63.

51. Chen D, Wang X, Huang J, et al. CDKN1B Mediates Apoptosis of Neuronal Cells and Inflammation Induced by Oxyhemoglobin via miR-502-5p After Subarachnoid Hemorrhage. J Mol Neurosci 2020;70:1073-80.

52. Razavipour SF, Harikumar KB, Slingerland JM. p27 as a transcriptional regulator: New roles in development and cancer. Cancer Res 2020;80:3451-8.

53. Peng M, Wang J, Tian Z, et al. Autophagy-mediated Mir6981 degradation exhibits CDKN1B promotion of PHLPP1 protein translation. Autophagy 2019;15:1523-38. 
54. Pan WK, Li P, Guo ZT, et al. Propranolol induces regression of hemangioma cells via the down-regulation of the PI3K/Akt/eNOS/VEGF pathway. Pediatr Blood Cancer 2015;62:1414-20.

55. Hu XH, Zhao ZX, Dai J, et al. MicroRNA-221 regulates osteosarcoma cell proliferation, apoptosis, migration, and invasion by targeting CDKN1B/p27. J Cell Biochem 2019; 120:4665-74.

56. Yang K, Chen Y, Cui Z, et al. MicroRNA-222-3p participates in the development of oral squamous cell carcinoma by targeting CDKN1B. J Oral Pathol Med 2020;49:621-9.

57. Liu J, Zhang D, Mi X, et al. p27 suppresses arseniteinduced Hsp27/Hsp70 expression through inhibiting JNK2/c-Jun- and HSF-1-dependent pathways. J Biol Chem 2010;285:26058-65.

58. Wander SA, Zhao D, Slingerland JM. p27: a barometer of

Cite this article as: Wang QZ, Zhao ZL, Liu C, Zheng JW. Exosome-derived miR-196b-5p facilitates intercellular interaction in infantile hemangioma via down-regulating CDKN1B. Ann Transl Med 2021;9(5):394. doi: 10.21037/atm-206456 signaling deregulation and potential predictor of response to targeted therapies. Clin Cancer Res 2011;17:12-8.

59. Nicoli S, Knyphausen CP, Zhu LJ, et al. miR-221 is required for endothelial tip cell behaviors during vascular development. Dev Cell 2012;22:418-29.

60. Lee S, Bui Nguyen TM, Kovalenko D, et al. Sprouty1 inhibits angiogenesis in association with up-regulation of p21 and p27. Mol Cell Biochem 2010;338:255-61.

61. Panopoulou E, Murphy C, Rasmussen H, et al. Activin A suppresses neuroblastoma xenograft tumor growth via antimitotic and antiangiogenic mechanisms. Cancer Res 2005;65:1877-86.

62. Hu H, Xu H, Lu F, et al. Exosome-Derived miR-4865 p Regulates Cell Cycle, Proliferation and Metastasis in Lung Adenocarcinoma via Targeting NEK2. Front Bioeng Biotechnol 2020;8:259. 